\title{
Semantic feature extraction method for hyperspectral crop classification
}

\author{
M. C. Girish Babu' ${ }^{1}$ Padma M. C. ${ }^{2}$ \\ ${ }^{1}$ Assistant Professor Department of Computer Science \& Engineering, PES College of Engineering, Karnataka, India \\ ${ }^{2}$ Professor, Department of Computer Science \& Engineering, PES College of Engineering, Karnataka, India
}

\section{Article Info \\ Article history: \\ Received Jan 19, 2021 \\ Revised Jun 7, 2021 \\ Accepted Jun 14, 2021 \\ Keywords: \\ Crop classification \\ Deep learning \\ Dimension reduction \\ Feature extraction \\ Feature selection \\ Hyperspectral image \\ Machine learning}

\begin{abstract}
Hyperspectral imaging (HSI) is composed of several hundred of narrow bands (NB) with high spectral correlation and is widely used in crop classification; thus, induces time and space complexity, resulting in high computational overhead and Hughes phenomenon in processing these images. Dimensional reduction technique such as band selection and feature extraction play an important part in enhancing performance of hyperspectral image classification. However, existing method are not efficient when put forth in noisy and mixed pixel environment with dynamic illumination and climatic condition. Here the proposed Sematic Feature Representation based HSI (SFR-HSI) crop classification method first employ Image Fusion (IF) method for finding meaningful features from raw HSI spectrally. Second, to extract inherent features that keeps spatially meaningful representation of different crops by eliminating shading elements. Then, the meaningful feature set are used for training using Support vector machine (SVM). Experiment outcome shows proposed HSI crop classification model achieves much better accuracies and Kappa coefficient performance.
\end{abstract}

This is an open access article under the CC BY-SA license.

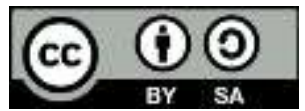

\section{Corresponding Author:}

M. C. Girish Babu

Assistant Professor, Department of Computer Science and Engineering

PES College of Engineering

Mandya, Karnataka, India

Email: girishbabuphd@gmail.com

\section{INTRODUCTION}

Hyperspectral imaging, like other spectral imaging, collects and processes information across the electromagnetic spectrum, usually in visible, near infrared, and short-wave infrared wavelengths. Recently, with the development of hyperspectral sensors, it has become possible to go beyond traditional RGB images and capture hundreds of spectral bands sampled with narrow wavelength intervals.Therefore,taking advantage of contiguous narrow bands, these HSI sensors enabled the analysis of the chemical properties of scene materials remotely for the purpose of detection, identification and chemical composition study of objects in a particular area. Hence, HSI captured using airborne sensor and earth observation satellites have been increasingly very helpful for urban planning, environment monitoring, and agriculture environment. However, this research work predominantly focuses on agriculture domain. This is because by year 2050 the population is expected to touch 9.6 billion [1]. Along with, the worldwide food demand is expanding, and in this way, the accessibility of exact, convenient, and timely data about agricultural on a neighborhood and also on worldwide [2], is fundamental [3] for guaranteeing that an increasing population can be served. So as to address the issues of unpredictability of the food market or meeting country food security, Hyperspectral imaging technique give a wide scope of prospects to measure these difficulties [2], [4]. 
Crop identification is one of the main element of agriculture crop monitoring by utilizing HSI obtained from satellite imagery. Crop mapping through HSI classification aid in making various decision making in agriculture environment such as yield forecasting, crop area assessment, and various other crop management applications [5], [6]. Accurate crop mapping is very important and impact crop identification applications. Nonetheless, limitation that exist in crop identification using HSI must be addressed [7]. First, the possible high dimension size of input HSI data is still an open issue. Second, HSI data resemble high similarities of textures, shapes and spectral signatures among different crop. Lastly, the presence of noise in HSI significantly impact the accuracies of existing HSI crop classification model.

The spectral resolutions have been significantly enhanced with growth of HSI methodologies. analyzing HSI is difficult. Further, considering the behaviour of HSI there exist higher correlation among neighboring pixel and spectral band sets [8]-[10]. Then, because of high dimensional nature of hyperspectral image there is high probability of increase in space, time and computational Thus, overhead leading to wellknown Hughes phenomenon [11]. Hence, reducing dimension of redundant feature in HSI is primarily necessary for processing hyperspectral image. Feature selection/Band Selection [12]-[14] is one of the efficient dimension reduction technique that remove redundant features. The basic notion of band selection methodologies is to choose most efficient band subset that is composed of information within original bands. On the other side, the feature extraction methodologies [15] reduces dimension in accordance with complex feature transformation. Thus, feature selection methods are easier to understand and can be used in practical purpose. The feature selection method can choose bands only within hyperspectral images. On the other side, the feature extraction method can utilize the HSI band sets for generating better discriminating feature sets. In Laplacian discriminant analysis of hyperspectral imagery [15], presented joint feature selection and feature extraction methodologies for hyperspectral image classification. Similar to [15] this paper focuses on developing the dimensionality reduction technique by extracting semantically useful feature set from raw hyperspectral image.

Numerous types of hyperspectral image dimensional reduction methodologies that includes both supervised and unsupervised technique, has been presented in recent time. This work focuses on supervised methodologies such as linear discriminant analysis [16], and also unsupervised methods such as independent component analysis (ICA) [17] and principal component analysis (PCA) [18]. From analysis it is seen that principal component analysis methodologies extract much better feature when compared with other methodologies. The PCA feature extraction technique can guarantee that more relevant feature of HSI can be kept with minimal size of useful principal components. On the other side, independent component analysis based methodologies can guarantee that the transformed components are independent as much as conceivable. Nonetheless, ICA based methodologies induce high computational overhead due to its complex computation. Further, the ICA methodologies does not consider spatial context information for extracting feature as they treat and process each pixel independently. Majority of existing HSI dimension reduction technique cannot directly utilize spatial information of hyperspectral images and feature extraction method uses only spectral information of every individual pixel which is stated to be efficient for enhancing the classification accuracies [19], [20]. The earth environment is composed of different types of corps such as soil, wheat, corn etc. hyperspectral image classification method aims at identifying these meaningful representation of crops. Thus, it is necessary to extract meaningful spatial feature sets-

Therefore, for extracting meaningful spatial information from hyperspectral image that composed of mixed pixel, it is important to capture inherent properties of physical characteristics of different objects. Thus by extracting spatial information from adjacent object can aid in increasing the accuracy of differentiating different crops present in earth environment. The inherent hyperspectral image are inherent feature set and shading component. Extracting these component plays very important role in classification task and at the same time it is challenging to extract these component from single hyperspectral image. This is because inherent feature sets relies on object features of the earth and varies with respect to illumination and climate condition. Thus, for extracting more meaningful spatial feature of different crop, this work present an efficient inherent feature extraction method namely SFR-HSI that keeps spatially meaningful representation of different crops. Further, for reducing the hyperspectral band dimension that is efficient against noise and mixed pixel environment, this work present an dimension reduction technique using fusion technique [21]. Lastly, dimensionally reduced feature sets are trained using multi-class support vector machine (SVM). Using SVM aid in training model with less training sample and can achieve good precision with minimal computation complexity when compared with deep learning based method [22]-[26].

The manuscript is articulated as described below. The proposed hyperspectral image crops classification model is presented in section II. The section III discusses about the result attained by proposed SFR-HSI crop classification model over various existing HSI crop classification model. In last section the significance of proposed SFR-HSI crop classification model is concluded with future direction of research work. 


\section{SEMANTIC FEATURE REPRESENTATION FOR HYPERSPECTRAL CROP CLASSIFICATION}

This work present an efficient crop classification method namely SFR-HSI using hyperspectral image. The block diagram of proposed crop classification technique is shown in Figure 1. The proposed SFRHSI hyperspectral crop classification is described in Algorithm 1. The first phase of hyperspectral crop classification is the feature reduction. For identifying useful feature sets from raw hyperspectral image, this work uses dimensionality reduction technique for enhancing classification accuracy.

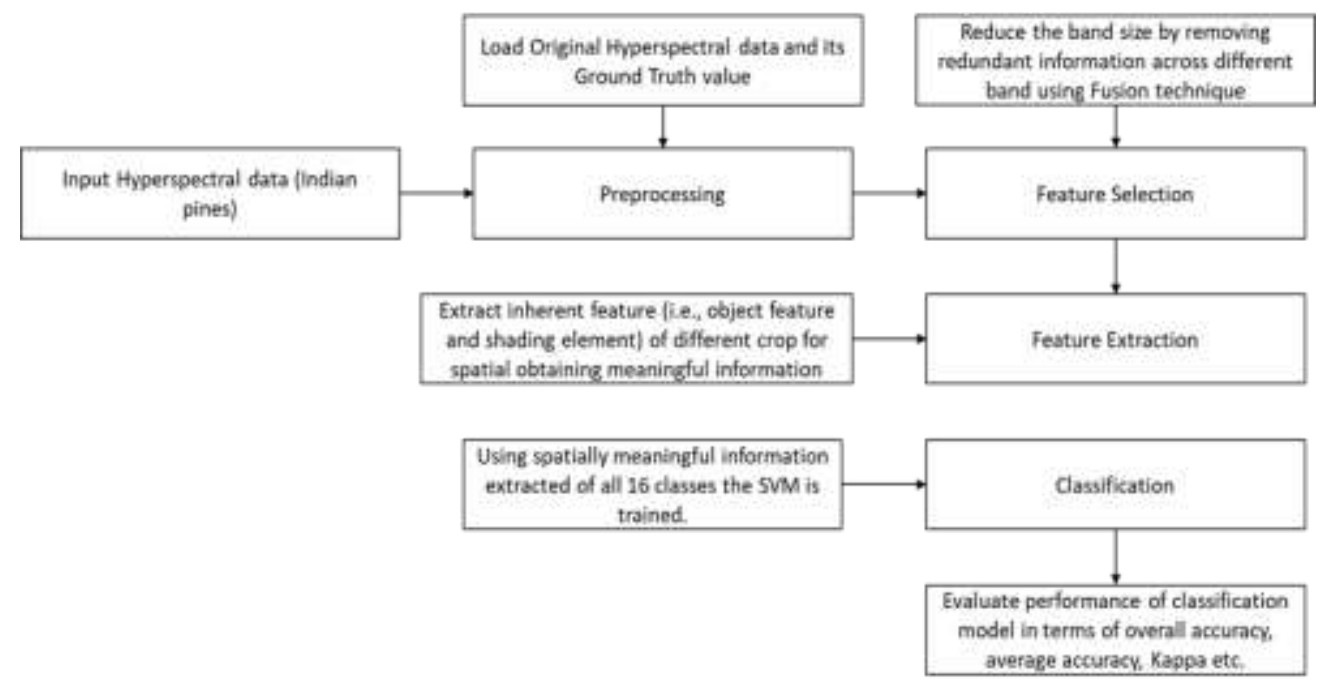

Figure 1. Block diagram of proposed SFR-HSI crop classification technique

Let us consider there are $K$ bands in a raw hyperspectral image, this work reduces the $K$ bands to $Q$ bands. The band $K$ is segmented into $Q$ sub-group sets. Then, mean of these sub-group sets is computed. For obtaining dimensionality reduced hyperspectral image feature most of the existing method used PCA. Using PCA can guarantee of preserving hyperspectral image features in a smaller quantity of useful principal components. However, using PCA cannot assure that the spectrally useful feature is retained. For addressing, the dimensionality reduced hyperspectral image feature is obtained by using fusion technique [21] method as (1)

$$
J^{l}=\frac{\sum_{m=(l-1) n+1}^{l n} J^{m}}{n}, l=\left\lfloor\frac{K}{Q}\right\rfloor,
$$

where $l$ and $m$ depicts the band indices, $m$ depicts the $m^{\text {th }}$ dimension of actual spectral band $(S B), l$ depicts the $l^{\text {th }}$ dimension of the reduced $S B, n$ depicts each sub-group band size, $[\cdot]$ rounds $\cdot$ to the closest value toward negative infinity, and $J^{m}$ depicts the redundant information and noisy pixel of each band sets. The (1) is used in eliminating redundant information and noisy pixels from each subgroup.

Algorithm 1. Proposed SFR-HSI Hyperspectral crop classification using dimension reduction and machine learning technique.

Input. Hyperspectral image collected from satellite $J \in S^{s * d * K}$ with $s * d$ pixels by $K$ bands.

Output. A classified outcome (i.e., labelled Hyperspectral image $Z$ ).

Step 1. Start.

Step 2. Dimension reduction of $K$ bands to $Q$ using Eq. (1).

Step 3. For $j \in\{1, Q\}$ do

Step 4. By iterating Eq. (5), the $J^{j}$ is decomposed into $S^{j}$ and $T^{j}$.

Step 5. End for.

Step 6. Vectorise $S$ to $Y=\left\{y_{1}, y_{2}, \ldots, y_{o}\right\} \in S^{Q * o}$ where $o=s * d$ for assuring each pixel can be represented as a $d$-dimensional data point.

Step 7. For obtaining labels $z \in S^{o}$ support vector machine learning algorithm is utilized.

Step 8. Reconstruct $Z$ to be a hyperspectral image $Z \in S^{Q * d}$.

Step 9. Stop 
Further, it is important to extract inherent properties (i.e., the minimum information required to hold the relevant information in any given image with mixed pixel is firmly known as the inherent properties) of hyperspectral image. The inherent feature sets rely on object (i.e., surface) features of the earth. These features are inherent with respect to illumination and climate condition. This work presents an efficient method for extracting inherent feature sets and eliminate the shading component in hyperspectral image to obtain semantically (i.e., meaningful) spatial information. Let $J \in S^{s * d}$ depict the intensity parameter of a hyperspectral image, $S \in S^{s * d}$ depicts its inherent feature set element and $T \in S^{s * d}$ depicts its inherent feature sets shading element. The hyperspectral image for a pixel $q$ is represented as a pixel-wise multiplicative of inherent feature set elements using (2)

$$
J_{q}=S_{q} T_{q}
$$

where $q$ depicts the pixel indices.

From (2), it is seen that a hyperspectral image is composed two inherent feature set elements, but still, it is difficult to solve the issue with two unknown parameters (i.e., $S_{q}$ and $T_{q}$ ) with one known value (i.e., $J_{q}$ ). In general, the reflectance is constant within each region and along edges has a sharp change. Further, it is seen as changes in intensity parameter will resemble a reflectance change and pixel with identical intensity parameter will resemble identical reflectance outcomes. Thus, $S_{q}$ is obtained using (3)

$$
S_{q}=\sum_{r \in \mathcal{O}(q)} b_{q r} S_{r}
$$

where $q$ and $r$ depicts pixel indexes, $b_{q r}$ depicts a feature sets component of affinity matrix (AM). The matrix depicting a pairwise similarities (PWS) among $J_{q}$ and $J_{r}$, and $O(q)$ depicts corresponding (i.e., neighbour) pixel $q$. The neighboring pixel are generally a Gaussian window $(G W)$ which can be obtained using (4)

$$
G W=\exp \left(-\frac{\|q-r\|_{2}^{2}}{2 \sigma^{2}}\right)
$$

and the size of it is established using $\sigma$. Further, defining affinity graph (AG) play very essential part in semantically extracting inherent characteristics. Using (2) and (4), we can establish the linear properties of these to (5)

$$
\left\{S_{r}=\sum_{r \in \mathcal{O}(q)} b_{q r} S_{r}, \widetilde{T}_{q}=\frac{1}{J_{q}} S_{r},\right.
$$

where $\widetilde{T}_{q}=\frac{1}{J_{q}}$. After obtaining the approximated outcome of $S_{r}$ and $T_{q}$, we can establish every pixel reflectance value (i.e., physical properties) of every object, where shading properties is not related with respect to semantic feature sets properties. Using inherent feature sets we can preserve the intrinsic properties of mixed pixel and eliminate the useless spatial information presented in shading element. Thus, we will get a spatially meaningful information of each objects/crop.

Lastly, for classification of optimized hyperspectral feature sets this work considered pixel wise SVM classification method. Support Vector Machine, also known as SVM, is popular for solving problems about classification, detection and regression. In support vector machine, the model constructs a hyperplane or set of hyperplanes in higher dimension space. From, [27] the structure of SVM is obtaitned as (6)

$$
\underset{W, b, \varphi}{\operatorname{argmax}} \frac{1}{2}\|W\|^{2}+C \sum_{n=1}^{N} \varphi_{n} \text { s.t } t_{n}\left(W^{T} \phi(X)+b\right) \geq 1-\varphi_{n ;} \varphi_{n} \geq 0 ; n=1,2, \ldots, N
$$

where $\phi(X)$ is transformed feature space and the variable $C$ is the regularization parameter to control the tradeoff between margin and the tolerance of misclassification. Using SVM model [27], can bring good tradeoff between margin and the tolerance of misclassification. In addition, in the dataset is non-linearly separable, applying kernel trick can transform them to higher linear dimension, such as Gaussian Kernel. Since the SVM problem is a convex optimization problem, the model can always obtain a global optimum from the model. With the optimal decision boundary, the mode can use it to classify hyperspectral dataset into different labels. The proposed hyperspectral image classification model attains better accuracy when compared with existing hyperspectral image classification model which is experimentally shown below. 


\section{RESULTS AND DISCUSSIONS}

This section discusses about the result achieved using proposed improved dimension reduction based hyperspectral crop classification method adopting machine learning technique. Further, the result obtained is compared with existing hyperspectral crop classification adopting machine and deep learning techniques. For carrying out experiment the data is collected using AVIRIS sensor [28]. This work selected the Indian Pines dataset for evaluating crop classification model because it contains of two-third of agriculture scene and rest is composed of forest and other vegetation crops. The dataset is composed of 16 classes which is described in Table 1 [29]. The Indian Pines dataset that composed of 220 spectral reflectance bands with wavelength range of $0.4-2.5 * 10^{-6}$ meters where twenty water absorption hyperspectral bands ranging from 104-108, 150-163, and 220 are removed and used for experiment analysis similar to [21], and [29]. The Indian pine image captured using AVIRIS sensor and its ground truth value is shown in Figure 2 (a), and Figure 2 (b), respectively. The dataset is composed of 10249 samples. Experiment are conducted to evaluate the performance of proposed hyperspectral crop classification model over various existing hyperspectral crop classification ranging from feature extraction method, feature selection, machine learning and deep learning method. The performance is evaluated in terms of overall accuracy (OA), average accuracy (AA), and Kappa coefficient (K). The overall accuracies performance depicts the correctly classified feature over total test feature. Average accuracies represents, the average of each individual classes. Kappa coefficient involves both commission and omission loses and provides an efficient information of the classification model robustness performances. For all the performance metric, higher the value depicts superior classification outcomes.

Table 1. Total samples of each classes of Indian Pines HSI data

\begin{tabular}{ccc}
\hline Number & Classes & Total Samples \\
\hline 1$)$ & Alfalfa & 46 \\
$2)$ & Corn notill & 1428 \\
$3)$ & Corn mintill & 830 \\
$4)$ & Corn & 237 \\
$5)$ & Grass pasture & 483 \\
$6)$ & Grass trees & 730 \\
$7)$ & Grass pasture moved & 28 \\
$8)$ & Hay windrowed & 478 \\
$9)$ & Oats & 20 \\
$10)$ & Soybean notill & 972 \\
$11)$ & Soybean mintill & 2455 \\
$12)$ & Soybean clean & 593 \\
$13)$ & wheat & 205 \\
$14)$ & woods & 1265 \\
$15)$ & Buildings Grass Trees Drives & 386 \\
$16)$ & Stone Steel Towers & 93 \\
\hline
\end{tabular}

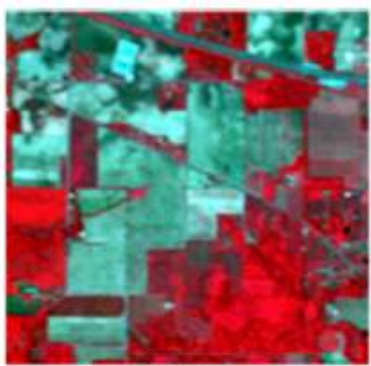

(a)

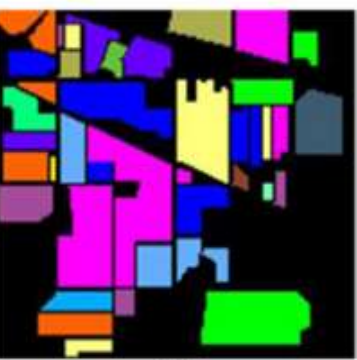

(b)

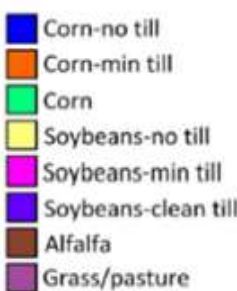

Grass/pasture

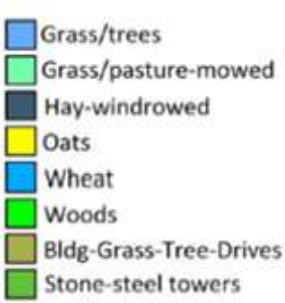

(c)

Figure 2. Indian Pines data set; (a) Indian Pines HIS, (b) Ground truth information of Indian pine HSI and (c) Corresponding label information of different crop of the Indian Pines HSI

\subsection{Feature extraction}

The Figure 2 (a) shows the actual satellite image captured of Indian pine data. Figure 2 (b) shows the reference image i.e., the ground truth value of Indian pine image. From Figure 2 (c) it can be seen there are different kind of crop which is represented by different color. First, both actual and ground truth image value are obtained (i.e., loaded). Total there are 16 classes. Then, using ground truth value information of a particular crop the feature set are extracted in random manner from respective source image (Figure 2 (a)) which are later used for training purpose. In same way the feature set are extracted for all 16 classes in random manner by varying space and range parameters. Then, rest of image will be used for testing purpose. Prior to performing feature extraction, first the band size of hyperspectral data is reduced using Image fusion technique [21] by eliminating redundant features using neighboring pixel correlation. Then, for feature extraction corresponding crop (i.e., object) inherent features are extracted using absorption and reflectance properties by varying space and range parameter. That is each object with different pixel intensity will have different reflectance wavelength (this is because some object like water will absorb some portion of 
wavelength and some object doesn't absorb). From Figure 2 it can be seen the edges of each crop is composed of different shading element (noise) and are separated by black color. These shading components are extracted using intensity parameter (i.e., reflectance properties of object). In our work shading element will have much lesser search space value when compared with intensity value. Then, these features are compared with object inherent properties, if it varies, they are eliminated or else feature are retained.

\subsection{Classification}

A The, feature set extracted of all 16 classes/crops are trained using SVM classification method. For evaluating performance this work considered 10-fold cross validation. The classification outcome is evaluated in terms of overall accuracy, Kapa Coefficient, and average accuracy.

\subsection{Experiment parameter optimization for hyperspectral crop classification}

The factors such as illumination, shading, and noise, the spectral features of different land covers in natural scenes are usually subject to some degree of distortion. In general, the reflectance is constant within each region and along edges has a sharp change. Further, it is seen as changes in intensity parameter will resemble a reflectance change and pixel with identical intensity parameter will resemble identical reflectance outcomes. Thus, using affinity pairwise similarity matrix among neighborhood pixels we can semantically extract inherent characteristics. Then we can establish every pixel reflectance value (i.e., physical properties) of every object, where shading properties is not related with respect to semantic feature sets properties. Using inherent feature sets we can preserve the intrinsic properties of mixed pixel and eliminate the useless spatial information presented in shading element. Thus, we will get a spatially meaningful information of each objects/crop. An important thing to be noted is the pairwise similarity is computed using two distance metrics such as range distance among intensity $j_{q}$ and intensity $j_{r}$ and other metric is space distance among a pixel $q$ and its adjacent pixel $r$. Thus, we have varied the parameter and experiment is conducted to evaluate its influence on classification accuracy. First, we have evaluated the influence of space parameter by fixing range parameter. In similar manner the influence of range parameter is analyzed by keeping space parameter fixed. Further, it is noticed keeping higher value of range parameter affect the classification accuracy. This is because using image fusion based recursive filter with higher range vale will neglect useful edge features. Thus, some classes are not identified properly. On similar terms keeping both space and range to small value will not yield good outcomes. This is because during feature extraction procedure very a smaller number of local spatial information would have been considered. Finally, from empirical analysis in work the space and range parameter are set to 200 and 0.1 respectively.

\subsection{Comparative analysis of SFR-HSI over spatial-spectral feature-based and machine leaning-based HSI crop classification model}

This section presents performance evaluation according to parameter defined in [30], [28]. In [30] presented a multiscale joint collaborative representation with locally adaptive dictionary (MLJCRC). For training model from each class, $10 \%$ of the samples are selected in random manner and remaining samples are used for testing purpose. The classification accuracy attained by proposed hyperspectral image classification performance over existing, MLJCRC classification model [30] is graphically shown in Figure 3. It can be seen that SVM model [30] attain an OA, AA, and Kappa coefficient performance of 82.79\%, $77.83 \%$, and $80.33 \%$, respectively. LJCRC model [30] an OA, AA, and Kappa coefficient performance of 95.18\%, 91.22\%, and 94.5\%, respectively. MLJCRC model [30] attain an OA, AA, and Kappa coefficient performance of $96.8 \%, 91.63 \%$, and $96.34 \%$, respectively. The proposed model attains an OA, AA, and Kappa coefficient performance of $97.98 \%, 97.7 \%$, and $97.69 \%$, respectively. From overall result attained it can be seen the proposed SFR-HSI model attain much better classification performance when compared with other state-of-art spatial and machine learning based classification algorithm.

\subsection{Comparative analysis over deep learning based hyperspectral crop classification model}

This section presents performance evaluation according to parameter defined in [24]. In [24] presented a Shorten Spatial-spectral RNN with Parallel-GRU (St-SS-pGRU1) for hyperspectral crop classification. For training model 1765 samples are selected in random manner and remaining samples are used for testing purpose. The calcification accuracy attained by proposed hyperspectral image classification performance over existing, MLJCRC classification model [24] is graphically shown in Figure 4. It can be seen that St-SS-GRU model [24] attain an OA performance of 87.16\%. St-SS-pGRU model [24] an OA performance of $90.35 \%$. GRU model [24] attain an OA of $77.01 \%$. The proposed model attains an OA performance of $98.47 \%$. From overall result attained it can be seen the proposed model attain much better classification performance when compared with other deep learning classification algorithm. 


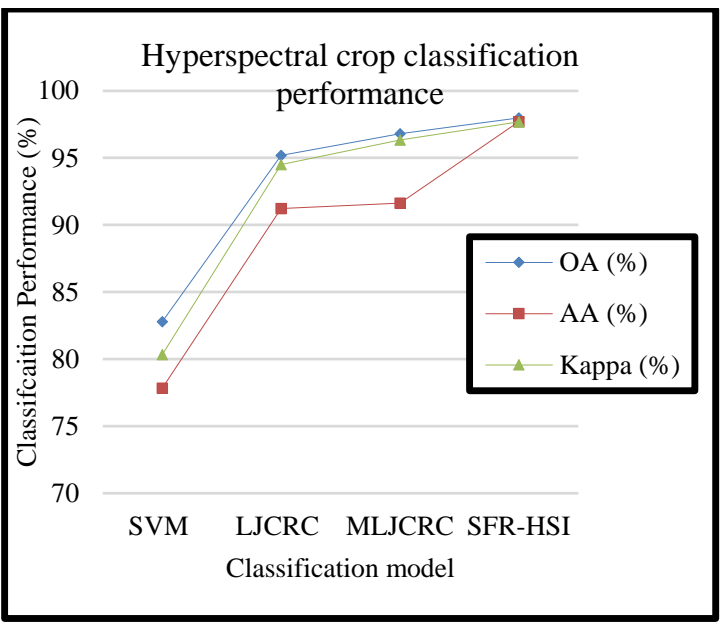

Figure 3. The classification outcome attained by proposed SFR-HSI classification model over existing HSI classification method

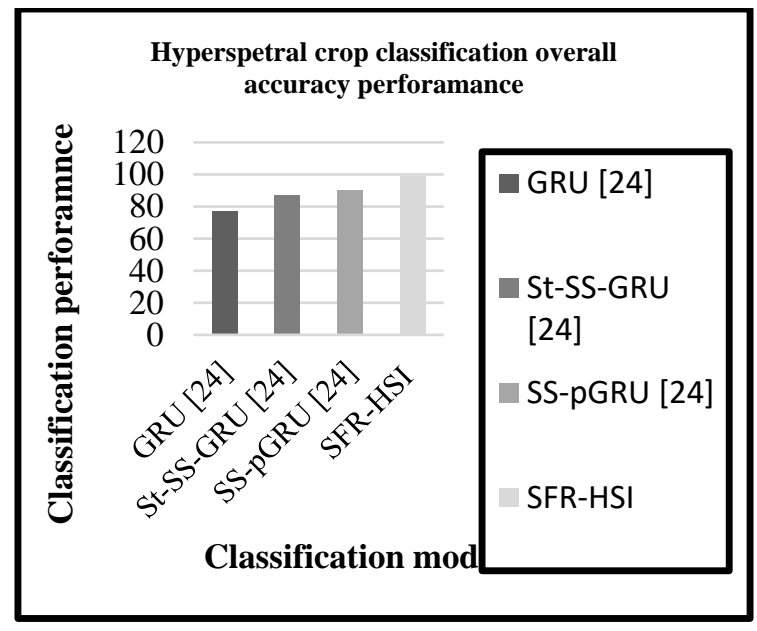

Figure 4. The classification outcome attained by proposed SFR-HSI classification model over existing deep learning HSI classification method

\section{CONCLUSION}

In this work, we have conduced deep rooted analysis of various hyperspectral crop classification model. From extensive analysis, it is noted that crop identification and classification using satellite images is challenging. Various methodologies for feature extraction, feature selection, and classification model using machine learning and deep learning model by exploiting spatial and spectral information has been presented for crop classification. Each model has its own advantages and disadvantage. Existing dimensional reduction technique features cannot assure that the spectrally useful features are retained. For improving classification accuracy considering noisy and mixed pixel environment, existing method adopted deep learning approach rather than machine learning model. Thus, these model induces high computation overhead and requires large number of labeled training sample. Therefore it is important to bring good tradeoff among classification accuracy and computational complexity. For addressing these issues, this work presented an efficient dimensional reduction technique that retain meaningful spatial information. Along with, can address noisy and mixed pixel problem affecting crop classification by learning (i.e., capturing) inherent properties of physical characteristics of different objects under different illumination and climatic condition. The proposed hyperspectral crop classification model is efficient as it requires very less training data when compared with deep learning based crop classification method. Experiment are conducted on standard dataset. The existing MLJCRC obtain an OA, AA, and Kappa coefficient performance of 96.8\%, 91.63\%, and 96.34\% which is much higher than other existing crop classification model. The proposed model attain much superior OA, AA, and Kappa coefficient performance of 97.98\%, 97.7\%, and 97.69\% which better than MLJCRC. Further, experiment are conducted for comparing proposed model existing deep learning based models. The SSpGRU model attain an OA performance of 90.35 which is much higher than other existing crop classification model. The proposed model attain much superior OA performance of $98.47 \%$ which better than SS-pGRU. Thus, proposed model is much efficient when compared with existing hyperspectral image classification. Future work would consider inducing artificial noise into hyperspectral image and see how the model perform. Further, refine the dimensional reduction method for obtaining more efficient feature extraction and also develop an improved classification algorithm that learns mixed pixel more efficiently.

\section{REFERENCES}

[1] P. Gerland et al., "World population stabilization unlikely this century," Science, vol. 346, no. 6206, pp. 234-237, 2014, doi: $10.1126 /$ science. 1257469 .

[2] A. K. Whitcraft, I. B.-Reshef and C. O. Justice, "A framework for defining spatially explicit earth observation requirements for a global agricultural monitoring initiative (GEOGLAM)," Remote Sens, vol. 7, pp. 1461-1481, 2015, doi: 10.3390/rs70201461.

[3] J. Fuhrer and P. J. Gregory, "Climate Change Impact and Adaptation in Agricultural Systems: Soil Ecosystem Management in Sustainable Agriculture," Wallingford, CT, USA: CABI, 2014.

[4] A. Mahalanobis, B. V. V. Kumar and S. R. Sims, "Distance-classifier correlation filters for multiclass target recognition," Applied Optics, vol. 35, no. 17, pp. 3127-3133, 2013, doi: 10.1364/AO.35.003127. 
[5] F. Low, U. Michel, S. Dech and C. Conrad, "Impact of feature selection on the accuracy and spatial uncertainty of per-field crop classification using support vector machines," ISPRS J. Photogrammetry Remote Sens., vol. 85, pp. 102-119, 2013, doi: 10.1016/j.isprsjprs.2013.08.007.

[6] S. Murmu and S. Biswas, "Application of fuzzy logic and neural network in crop classification: A review," Aquatic Procedia, vol. 4, pp. 1203-1210, 2015, doi: 10.1016/j.aqpro.2015.02.153.

[7] K. Tatsumi, Y. Yamashiki, M. A. C. Torres and C. L. R. Taipe, "Crop classification of upland fields using Random forest of time-series Landsat 7 ETM+ data," Comput. Electron. Agriculture, vol. 115, pp. 171-179, 2015, doi: 10.1016/j.compag.2015.05.001.

[8] M. Gong, M. Zhang and Y. Yuan, "Unsupervised Band Selection Based on Evolutionary Multiobjective Optimization for Hyperspectral Images," in IEEE Transactions on Geoscience and Remote Sensing, vol. 54, no. 1, pp. 544-557, Jan. 2016, doi: 10.1109/TGRS.2015.2461653.

[9] W. Sun and Q. Du, "Graph-Regularized Fast and Robust Principal Component Analysis for Hyperspectral Band Selection," in IEEE Transactions on Geoscience and Remote Sensing, vol. 56, no. 6, pp. 3185-3195, June 2018, doi: 10.1109/TGRS.2018.2794443.

[10] H. Zhai, H. Zhang, L. Zhang and P. Li, "Laplacian-Regularized Low-Rank Subspace Clustering for Hyperspectral Image Band Selection," in IEEE Transactions on Geoscience and Remote Sensing, vol. 57, no. 3, pp. 1723-1740, March 2019, doi: 10.1109/TGRS.2018.2868796.

[11] F. Melgani and L. Bruzzone, "Classification of hyperspectral remote sensing images with support vector machines," in IEEE Transactions on Geoscience and Remote Sensing, vol. 42, no. 8, pp. 1778-1790, Aug. 2004, doi: 10.1109/TGRS.2004.831865.

[12] M. Zhang, M. Gong and Y. Chan, "Hyperspectral band selection based on multi-objective optimization with high information and low redundancy," Applied Soft Computing, vol. 70, pp. 604-621, 2018, doi: 10.1016/j.asoc.2018.06.009.

[13] P. Hu, X. Liu, Y. Cai and Z. Cai, "Band Selection of Hyperspectral Images Using Multiobjective OptimizationBased Sparse Self-Representation," in IEEE Geoscience and Remote Sensing Letters, vol. 16, no. 3, pp. 452-456, March 2019, doi: 10.1109/LGRS.2018.2872540.

[14] Q. Wang, F. Zhang and X. Li, "Optimal Clustering Framework for Hyperspectral Band Selection," in IEEE Transactions on Geoscience and Remote Sensing, vol. 56, no. 10, pp. 5910-5922, Oct. 2018, doi: 10.1109/TGRS.2018.2828161.

[15] X. Jiang, X. Song, Y. Zhang, J. Jiang, J. Gao, and Z. Cai, "Laplacian regularized spatial-aware collaborative graph for discriminant analysis of hyperspectral imagery," Remote Sensing, vol. 11, no. 1, pp. 29, 2019.

[16] W. Liao, A. Pizurica, P. Scheunders, W. Philips and Y. Pi, "Semisupervised Local Discriminant Analysis for Feature Extraction in Hyperspectral Images," in IEEE Transactions on Geoscience and Remote Sensing, vol. 51, no. 1, pp. 184-198, Jan. 2013, doi: 10.1109/TGRS.2012.2200106.

[17] A. Villa, J. A. Benediktsson, J. Chanussot and C. Jutten, "Hyperspectral Image Classification with Independent Component Discriminant Analysis," in IEEE Transactions on Geoscience and Remote Sensing, vol. 49, no. 12, pp. 4865-4876, Dec. 2011, doi: 10.1109/TGRS.2011.2153861.

[18] S. Prasad and L. M. Bruce, "Limitations of Principal Components Analysis for Hyperspectral Target Recognition," in IEEE Geoscience and Remote Sensing Letters, vol. 5, no. 4, pp. 625-629, Oct. 2008, doi: 10.1109/LGRS.2008.2001282.

[19] L. Wang, J. Zhang, P. Liu, K.-K. R. Choo and F. Huang, "Spectral-spatial multi-feature-based deep learning for hyperspectral remote sensing image classification," Soft Computing, vol. 21, pp. 213-221, 2016, doi: 10.1007/s00500-016-2246-3.

[20] J. Jiang, C. Chen, Y. Yu, X. Jiang and J. Ma, "Spatial-Aware Collaborative Representation for Hyperspectral Remote Sensing Image Classification," in IEEE Geoscience and Remote Sensing Letters, vol. 14, no. 3, pp. 404408, March 2017, doi: 10.1109/LGRS.2016.2645708.

[21] X. Kang, S. Li and J. A. Benediktsson, "Feature Extraction of Hyperspectral Images with Image Fusion and Recursive Filtering," in IEEE Transactions on Geoscience and Remote Sensing, vol. 52, no. 6, pp. 3742-3752, June 2014, doi: 10.1109/TGRS.2013.2275613.

[22] A. Santara et al., "BASS Net: Band-Adaptive Spectral-Spatial Feature Learning Neural Network for Hyperspectral Image Classification," in IEEE Transactions on Geoscience and Remote Sensing, vol. 55, no. 9, pp. 5293-5301, Sept. 2017, doi: 10.1109/TGRS.2017.2705073.

[23] X. Cao, F. Zhou, L. Xu, D. Meng, Z. Xu and J. Paisley, "Hyperspectral Image Classification With Markov Random Fields and a Convolutional Neural Network," in IEEE Transactions on Image Processing, vol. 27, no. 5, pp. 23542367, May 2018, doi: 10.1109/TIP.2018.2799324.

[24] H. Luo, "Shorten Spatial-spectral RNN with Parallel-GRU for Hyperspectral Image Classification," arXiv:1810.12563v1, 2018.

[25] Y. Cai, X. Liu and Z. Cai, "BS-Nets: An End-to-End Framework for Band Selection of Hyperspectral Image," in IEEE Transactions on Geoscience and Remote Sensing, vol. 58, no. 3, pp. 1969-1984, March 2020, doi: 10.1109/TGRS.2019.2951433.

[26] Y. Liang, X. Zhao, A. J. X. Guo and F. Zhu, "Hyperspectral Image Classification With Deep Metric Learning and Conditional Random Field," in IEEE Geoscience and Remote Sensing Letters, vol. 17, no. 6, pp. 1042-1046, June 2020, doi: 10.1109/LGRS.2019.2939356.

[27] F. Li, K. Ergun, O. Kilinc and Y. Qiao, "Vegetation Classification Using Hyperspectral Images," in ECE228 and SIO209 Machine learning for physical applications, 2018. 
[28] L. Wang, Y. Feng, Y. Gao, Z. Wang and M. He, "Compressed Sensing Reconstruction of Hyperspectral Images Based on Spectral Unmixing," in IEEE Journal of Selected Topics in Applied Earth Observations and Remote Sensing, vol. 11, no. 4, pp. 1266-1284, April 2018, doi: 10.1109/JSTARS.2017.2787483.

[29] M. C. G. Babu and M. C. Padma, "A Efficient Solution for Classification of Crops using Hyper Spectral Satellite Images," International Journal of Innovative Technology and Exploring Engineering (IJITEE), vol. 9, no. 2, pp. 5204-5211, 2019, doi: 10.35940/ijitee.B7311.129219.

[30] J. Yang and J. Qian, "Hyperspectral Image Classification via Multiscale Joint Collaborative Representation With Locally Adaptive Dictionary," in IEEE Geoscience and Remote Sensing Letters, vol. 15, no. 1, pp. 112-116, Jan. 2018, doi: 10.1109/LGRS.2017.2776113.

\section{BIOGRAPHIES OF AUTHORS}

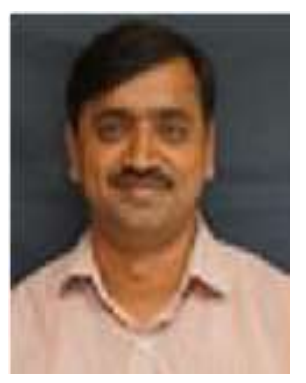

Girish Babu M C received the B. E Degree in Computer Science \& Engineering in 2004, M. Tech in 2009 from Visvesvaraya Technological University. He is research scholar in the field of Image processing at Visvesvaraya Technological University India.

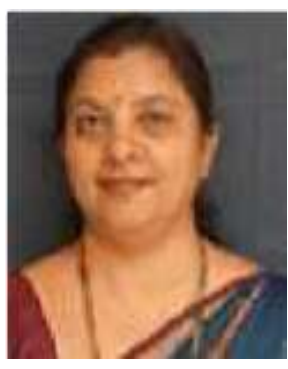

Dr. Padma M C received the B.E Degree in Computer Science \& Engineering in 1990, Msc (Tech) from University of Mysore, India in 2004 and the Phd Degree in Image Processing from Visvesvaraya Technological University in 2010. She has published more than 45 international/national Journals. Her main research area includes Natural Language Processing, Data mining, Image processing and Pattern recognition. 